\title{
O PRONTUÁRIO ELETRÔNICO DO PACIENTE NA PERSPECTIVA DAS RECOMENDAÇÕES DE USABILIDADE: PROPOSTA DE ORGANIZAÇÃO DA INFORMAÇÃO
}

\author{
REGISTRO ELECTRÓNICO DEL PACIENTE EN LA \\ PERSPECTIVA DE LAS RECOMENDACIONES DE \\ USABILIDAD: PONENCIA DE LA ORGANIZACIÓN DE LA \\ INFORMACIÓN EN EL
}

\author{
Tatiana Tissa Kawakami* \\ Rosane Alvares Lunardelli** \\ Fernando Luiz Vechiatto***
}

\begin{abstract}
RESUMO:
Introdução: dentre as diversas esferas de estudos existentes, ressalta-se a informação na área da Saúde. Mais especificamente, foca-se o prontuário eletrônico do paciente e questões relacionadas à sua organização informacional e usabilidade.

Objetivos: desenvolver proposta de recomendações de usabilidade aplicáveis ao prontuário eletrônico do paciente. Mais especificamente, identificar, mediante revisão de literatura especializada, recomendações de Usabilidade e desenvolver um checklist com recomendações de Usabilidade para o prontuário eletrônico do paciente.

Metodologia: o estudo é de finalidade básica e cunho teórico. Utilizou-se o método dedutivo de delineamento documental.

Resultados: elaboração de checklist com recomendações de Usabilidade aplicáveis ao prontuário.

Conclusão: recomendações de usabilidade podem ser utilizadas para aprimoramento do prontuário eletrônico do paciente. Ainda assim, destaca-se que conhecimentos do âmbito da Ciência da Informação devem ser considerados e somados, uma vez que grande parte de conteúdos relacionados à Usabilidade referem-se a aspectos operacionais e visuais da interface, não contemplando de maneira clara e direta a questão informacional.
\end{abstract}

Palavras-chave: Usabilidade. Prontuário Eletrônico do Paciente. Organização da Informação.

*Mestre em Ciência da Informação. E-mail: tissattk@gmail.com **Doutora em Estudos da Linguagem. E-mail: lunardelli@uel.br

***Doutor em Ciência da Informação. E-mail: vechiato2004@yahoo.com.br 
Tatiana Tissa Kawakami, Rosane Alvares Lunardelli, Fernando Luiz Vechiato O prontuário eletrônico do paciente na perspectiva das recomendações de usabilidade: proposta de organização da informação

\section{INTRODUÇÃO}

Ao se relacionar informação com a área da Saúde, uma das formas de registro de maior relevância é o prontuário eletrônico do paciente (PEP), complexo documento composto de conteúdos diversos, como receitas médicas, notas de evolução do paciente, exames clínicos, entre outros. Além da vasta gama de informações nele presentes, outro elemento que contribui para sua complexidade é o fato de esse ser instrumento de trabalho para profissionais de múltiplas áreas (médicos, enfermeiros, fisioterapeutas, nutricionistas, entre outros). Ademais, o prontuário do paciente pode ser utilizado como fonte de informação em outros contextos além da Saúde como, por exemplo, os âmbitos acadêmico (pesquisa) e jurídico (documento probatório).

Assim, sabendo de sua importância e aplicabilidade em diferentes áreas, além de considerar seu caráter sigiloso no que diz respeito às informações nele registradas, destaca-se a necessidade de estudos direcionados a esse dossiê que registra a passagem do paciente em uma instituição voltada à saúde. Nesse cenário acredita-se que a Usabilidade, que tem por enfoque investigar meios para otimizar a eficiência e eficácia de produtos/serviços, bem como a satisfação dos usuários, possa contribuir para investigações relacionadas à organização da informação nele contida.

Diante desse cenário, buscou-se desenvolver uma proposta de recomendações de usabilidade aplicáveis ao PEP.

Em linhas gerais o trabalho constitui-se em três partes: identificação e estudo de literatura especializada, análise das recomendações de usabilidade identificadas e, por fim, construção de checklist com recomendações de usabilidade aplicáveis ao PEP. A expectativa é a de que, a partir das recomendações propostas para o PEP, seja possível tornar maior a chance de sucesso em sua implantação e utilização, favorecendo o trabalho da equipe de profissionais da Saúde e beneficiando, assim, em última instância, mas não menos importante, o próprio paciente.

Acredita-se na potencial contribuição do presente estudo para a sociedade pautando-se na ideia de que a saúde configura-se como fator de caráter decisivo 
Tatiana Tissa Kawakami, Rosane Alvares Lunardelli, Fernando Luiz Vechiato O prontuário eletrônico do paciente na perspectiva das recomendações de usabilidade: proposta de organização da informação

para a sustentação, desenvolvimento e evolução do ser humano. Além disso, ao evidenciar a importância da informação na Saúde e suas possíveis implicações, espera-se ressaltar a capacidade da Ciência da Informação de contribuir de maneira relevante para a solução de problemas referentes à organização, à recuperação e ao acesso do grande fluxo informacional em contextos cruciais ao desenvolvimento da sociedade.

\section{PERCURSO METODOLÓGICO}

O presente estudo é de finalidade básica e cunho teórico, sendo dedutivo e de delineamento documental. Um dos fatores que influenciou o delineamento documental da pesquisa é o fato de essa ter como um de suas características "[...] não exigir contato com os sujeitos da pesquisa. É sabido que em muitos casos o contato com os sujeitos é difícil ou até mesmo impossível." (GIL, 2008, p. 46).

A questão da dificuldade de contato com o sujeito da pesquisa é certamente um fator delimitador presente neste estudo, uma vez que o acesso à equipe de profissionais que utilizam o PEP, bem como a autorização, por parte de instituições de Saúde, ao prontuário em si, é imbuído de diversos obstáculos. Essa dificuldade ocorre pelo fato de que, por se tratar de um documento com conteúdos confidenciais, as instituições apresentam grande resistência e uma série de restrições com relação a assuntos concernentes ao PEP.

Ademais, esta pesquisa é dedutiva, pois parte do geral para chegar ao específico (SANTOS, 2005). Nesse sentido, o estudo pautou-se nas recomendações gerais de Usabilidade propostas por Nielsen (1995), Jordan (1998), Shneiderman (1998), Rogers, Sharp e Preece (2013) e Norman (2006), as quais foram complementadas pelas perspectivas com foco na informação apresentadas por Morville e Rosenfeld (2006) e Ranganathan (2009), buscando assim delinear recomendações de Usabilidade específicas para o contexto do PEP.

Para a construção do aporte teórico, recorreu-se ao uso de literatura corrente e de referência, tendo sido consultados periódicos científicos (eletrônicos e impressos), livros, manuais e diferentes bases de dados como SciELO e Portal 
Tatiana Tissa Kawakami, Rosane Alvares Lunardelli, Fernando Luiz Vechiato O prontuário eletrônico do paciente na perspectiva das recomendações de usabilidade: proposta de organização da informação

CAPES. Foram consultadas tanto publicações nacionais quanto internacionais, sem demarcação temporal e linguística.

Uma vez realizado o levantamento de aporte teórico preliminar deu-se, conforme recomendado por Gil (2008), a leitura seletiva e analítica de conteúdos levando-se em conta os objetivos a serem alcançados na pesquisa. Por fim, a construção do checklist com recomendações de usabilidade aplicáveis ao PEP se deu por meio do entrelaçamento de conteúdos selecionados no referencial teórico.

\section{PRONTUÁRIO ELETRÔNICO DO PACIENTE}

O prontuário do paciente configura-se como "[...] um elemento crucial no atendimento à saúde dos indivíduos, devendo reunir a informação necessária para garantir a continuidade dos tratamentos prestados ao cliente/paciente." (MASSAD et al., 2003, p. 1). Ainda segundo os autores mencionados, o prontuário do paciente tem a finalidade de dar suporte aos processos de atenção à saúde provendo informações médicas e administrativas; registrar legalmente as ações médicas; servir como material para pesquisas (tanto no âmbito de estudos clínicos como no âmbito de ensino).

Com relação ao grau de importância desse dossiê, Weber (2010) enfatiza que a qualidade das informações no preenchimento desse documento é determinante não apenas para o bom atendimento do paciente, uma vez que também interfere diretamente em decisões de processos judiciais que englobem informações relacionadas a procedimentos de saúde. Por vezes, prontuários preenchidos de forma inadequada podem acarretar problemas judiciais para o profissional e para a instituição que prestou atendimentos.

O prontuário do paciente comporta diversos tipos de informação, dentre elas: ficha de identificação do paciente; histórico de medicamentos administrados; antecedentes pessoais; notas de evolução; relatórios de imagem; relatórios laboratoriais; formulários de consentimento e autorização de procedimentos. (WAGER; LEE; GLASER, 2009)

Logo, reconhecendo-se a complexidade de que se reveste o prontuário do paciente, "Cabe pensar essas novas composições da informação, a vida e a saúde, a partir da junção das informações e da tecnologia" (GONZALEZ DE 
Tatiana Tissa Kawakami, Rosane Alvares Lunardelli, Fernando Luiz Vechiato O prontuário eletrônico do paciente na perspectiva das recomendações de usabilidade: proposta de organização da informação

GOMEZ, 2013, p. 48). Nessa perspectiva, traz-se à luz o prontuário do paciente em seu formato eletrônico, o PEP. Esse é caracterizado por Lunardelli, Tonello e Molina (2014, p. 117) como "[...] documento único, de característica multimídia (composto por textos escritos, imagéticos), multifuncional (gerado e utilizado com diversas finalidades) e multiprofissional (registrado e consultado por vários profissionais da Área) [...]".

Com relação às diferenças entre o prontuário em papel e o prontuário eletrônico, nota-se que o último pode apresentar certas desvantagens, como o fato de depender de fatores como a disponibilidade de energia elétrica para ser utilizado, enquanto o prontuário em papel não apresenta esse tipo de fator limitador (CONSELHO REGIONAL DE MEDICINA DO DISTRITO FEDERAL, 2006).

Por outro lado, dentre os pontos positivos está a possibilidade de uma mesma informação ser trabalhada em diferentes partes e, se necessário, reutilizadas, evitando, assim, a redundância informacional (GALVÃO; RICARTE, 2011), o que não acontece com as informações registradas em suporte de papel. Além disso, o prontuário de papel "[...] pode apresentar partes ilegíveis ou incompletas, ocupar grande espaço nos arquivos." (CONSELHO REGIONAL DE MEDICINA DO DISTRITO FEDERAL, 2006, p. 48), problemas esses que podem ser evitados com a utilização do prontuário em versão eletrônica.

Jenal e Évora (2012) mencionam também que os dados gerados por meio de registros eletrônicos, por apresentarem maior facilidade de recuperação de informações em relação aos prontuários em papel, abrem maior possibilidade de utilizá-los como material para desenvolvimento de pesquisas históricas, sociológicas e arquivísticas.

Mesmo em face às inúmeras contribuições e facilidades potencialmente trazidas por meio da utilização do PEP, Bentes Pinto (2006) entende como relevante considerar também que este é um tipo de documentação bastante complexa, visto que sua produção ocorre de maneira "coletiva" (trabalho conjunto entre uma equipe multiprofissional de Saúde, o paciente e seus familiares). Galvão e Ricarte (2012, p. 15) evidenciam a expressiva gama de 
Tatiana Tissa Kawakami, Rosane Alvares Lunardelli, Fernando Luiz Vechiato O prontuário eletrônico do paciente na perspectiva das recomendações de usabilidade: proposta de organização da informação

pessoas envolvidas em processos relacionados com PEP ao citarem "[...] médicos, enfermeiros, fisioterapeutas, nutricionistas, psicólogos, odontólogos, assistentes sociais [...]." Nesse sentido, há de se levar em conta a existência, além das diferenças entre formações profissionais, de outros elementos como competências linguísticas, competências tecnológicas e fatores culturais, que também afetam o comportamento do indivíduo na utilização do PEP (GALVÃO; RICARTE, 2012).

Assim, diante do exposto, evidencia-se o caráter intrincado da implantação e utilização de PEP. No que tange aos obstáculos relacionados ao uso de prontuários em formato eletrônico, salienta-se que "[...] a instituição que implantar o PEP deve ter consciência do alto investimento em tecnologia e treinamento de pessoal, além da necessidade de propiciar condições para que seus usuários conheçam e incorporem novos paradigmas [...]" (LUNARDELLI; MOLINA; ALVES, 2009, p. 499).

Dentre os elementos apresentados, destaca-se a questão da capacitação da equipe/usuários. Nessa perspectiva, buscando identificar fatores relacionados à utilização de PEP bem sucedida, Canêo e Rondina (2014, p. 69) realizaram estudo no qual concluíram que são elementos decisivos "[...] treinamento intenso e adequado da equipe e à sua participação nas diversas etapas que precedem a implantação do sistema e à familiaridade dos usuários com o sistema implantado."

Jenal e Évora (2012, p. 180) corroboram tal perspectiva ao afirmarem: "Observa-se que o sucesso ou o fracasso [da implantação de um PEP] está intimamente ligado ao envolvimento dos usuários no planejamento e nas diversas fases de implantação do sistema [...]". Nesse contexto, tomando-se o usuário como parte indispensável no processo de desenvolvimento e implementação de um serviço de informação, introduz-se a questão da usabilidade.

\section{USABILIDADE}

No contexto da Ciência da Informação $(\mathrm{Cl})$, estudos centrados no usuário começaram a ser desenvolvidos na década de setenta, procurando, 
Tatiana Tissa Kawakami, Rosane Alvares Lunardelli, Fernando Luiz Vechiato O prontuário eletrônico do paciente na perspectiva das recomendações de usabilidade: proposta de organização da informação

dessa maneira, suprir as necessidades de compreender melhor o usuário dos serviços de informação e objetivando entender como este sujeito produz, faz uso e divulga informações (CARDOSO, 1996). Assim, nesse contexto, surge a questão da usabilidade e sua importância em interfaces de PEP.

Pode-se entender a usabilidade como um campo de estudos que busca garantir facilidades na utilização dos produtos e que provê aos usuários eficiência e uma experiência agradável (ROGERS; SHARP; PREECE, 2013), visando, assim, "[...] aumentar e melhorar o uso do produto" (CAMARGO; VIDOTTI, 2011, p. 6). De forma resumida, pode-se afirmar que, de acordo com Cybis, Betiol e Faust (2010, p. 16), "A essência da usabilidade é o acordo entre interface, usuário, tarefa e ambiente".

Jordan (1998) reflete a respeito da importância da usabilidade ao mencionar que ela possui o potencial de prevenir problemas relacionados ao âmbito financeiro, à segurança do usuário e a insatisfações/aborrecimentos durante o uso de determinada interface ou produto.

Inserindo-se as vantagens financeiras trazidas pela usabilidade no contexto da informação na Saúde, especificamente no PEP, compreende-se que um prontuário em formato eletrônico de fácil acesso é de interesse tanto para a empresa desenvolvedora de softwares quanto para a instituição que faz uso do PEP. Afinal, uma empresa que oferece um software de PEP que apresente maior usabilidade tem as chances de venda de seu produto favorecidas. Outrossim, uma instituição de cuidados com a saúde que adotar um PEP de fácil uso possibilitará que seus profissionais passem a trabalhar de maneira mais eficiente e eficaz, favorecendo, assim, tanto própria a instituição de cuidados com saúde, como também, em última instância, o indivíduo que está sendo tratado.

A questão da usabilidade relacionada à segurança dos usuários, no caso dos PEP, é extremamente relevante, uma vez que qualquer tipo de erro no registro de informações ou equívocos na utilização de funcionalidades do PEP pode acarretar sérios problemas para o trabalho da equipe de profissionais da saúde e, consequentemente, prejudicar o paciente. 
Tatiana Tissa Kawakami, Rosane Alvares Lunardelli, Fernando Luiz Vechiato O prontuário eletrônico do paciente na perspectiva das recomendações de usabilidade: proposta de organização da informação

Cabe observar que as dificuldades e frustrações ao utilizar um objeto ou interface estão, de certa forma, descumprindo com o propósito de sua própria existência, que deveria ser o de facilitar a execução de tarefas desempenhadas pelo homem (JORDAN, 1998). Nesse sentido, compreende-se que um PEP desprovido de usabilidade deixa de cumprir seu papel como ferramenta tecnológica catalizadora do processo de registro e utilização de informações inscritas nesse dossiê.

O prontuário eletrônico inevitavelmente evoca novas questões e problemas relacionados à usabilidade, sendo assim "[...] importante identificar como a adoção do PEP é percebida por profissionais de saúde que o utilizam, bem como analisar impactos desse sistema de prontuário no trabalho desses profissionais" (FARIAS et al., 2011, p. 1306).

Pesquisadores na área de Ciência da Informação, como Vechiato e Vidotti (2012), entre outros, apresentam a usabilidade como profundamente ligada às pesquisas em Interação Humano-Computador (IHC), ergonomia, Arquitetura da Informação (Al) e Comportamento Informacional. Cybis, Betiol e Faust (2010), que desenvolvem pesquisas no âmbito da Engenharia de Usabilidade, corroboram a perspectiva dos autores ao apontarem como áreas ligadas à Usabilidade a IHC e a Ergonomia. Já Barbosa e Silva (2010), pesquisadores de questões ligadas à $\mathrm{TIC}$, além de reconhecerem a $\mathrm{IHC}$ como domínio relacionado à usabilidade, destacam também a contribuição de estudos da área do Design. Batley (2007) e Rogers, Sharp e Preece (2013) reforçam a perspectiva de Barbosa e Silva (2010) ao apontarem o Design como campo de estudos relacionado à Usabilidade.

Embora nem todos os autores citados sejam pesquisadores direta e exclusivamente da área de usabilidade, todos eles promovem estudos que abordam questões relacionadas ao tema. Buscando-se investigar de maneira mais aprofundada a própria questão da usabilidade, faz-se pertinente a compreensão dos elementos anteriormente citados, destacando-se, no presente estudo, a Ergonomia, a IHC, a Al e o Design.

Nessa direção, traçaram-se relações entre pensamentos e recomendações de diferentes autores, sendo estes: Jakob Nielsen (1995), 
estudioso dos campos de IHC e Usabilidade; Patrick W. Jordan (1998), que desenvolve pesquisas integrando campos como psicologia, design, ergonomia e usabilidade; Ben Shneiderman (1998), que tece pesquisas relacionadas à IHC e design de interface, entre outros campos do conhecimento; Yvonne Rogers, Helen Sharp e Jennifer Preece (2013), que realizam investigações na área de design de interação e IHC; Donald A. Norman (2006), que combina em suas pesquisas conhecimentos das áreas de Design, Psicologia e Usabilidade; e, por fim, Peter Morville e Louis Rosenfeld (2006), pesquisadores do campo da Arquitetura da Informação (Al).

Nielsen (1995) propõe 10 recomendações (heurísticas) de usabilidade para o design de interface. Essas são pontuadas sumariamente a seguir:

1. Visibilidade do status do sistema: sempre informar o usuário sobre o que está acontecendo, por meio de feedback adequado e em tempo razoável.

2. Compatibilidade do sistema com o mundo real: "falar a língua dos usuários" com palavras, frases e conceitos familiares ao usuário. Seguir convenções do mundo real, fazendo com que a informação seja apresentada de forma lógica e natural.

3. Controle e liberdade do usuário: caso o usuário selecione funções do sistema por engano, fornecer uma alternativa para reparar o equivoco. Oferecer a possibilidade de desfazer e refazer.

4. Consistência e padrões: seguir padrões na plataforma para que o usuário não tenha que imaginar se diferentes palavras, situações ou ações apresentam o mesmo significado.

5. Prevenção de erros: ainda que oferecida mensagem de erro, é preferível um design que previna a ocorrência de problemas. Fornecer ao usuário a opção de confirmação da ação antes que ela seja finalizada.

6. Reconhecimento em vez de memorização: deve-se minimizar a carga de memória do usuário. Instruções de uso do sistema devem sempre ser de fácil alcance.

7. Flexibilidade e eficiência de uso: disponibilizar "aceleradores"/atalhos aos usuários mais experientes. Permitir a customização de ações frequentes.

8. Estética e design minimalista: os diálogos não devem conter informações irrelevantes ou raramente necessitadas.

9. Ajudar os usuários a reconhecer, diagnosticar e recuperar-se de erros: mensagens de erro devem ser objetivas, sem apresentação de códigos. Indicar precisamente qual o problema e oferecer uma solução de maneira construtiva. 
10. Ajuda e documentação: mesmo sendo preferível que o sistema possa ser utilizado sem o auxílio, disponibilizar ajuda e documentação de fácil acesso.

Nesse sentido, Jordan (1998) apresenta também dez recomendações voltadas à usabilidade de interfaces. Segue breve descrição de cada uma das recomendações:

1. Consistência: conceber um produto de maneira que tarefas semelhantes sejam executadas de maneira semelhante.

2. Compatibilidade: visa ao desenvolvimento de um produto de modo que sua forma de utilização seja compatível às expectativas dos usuários. Levar em conta os conhecimentos e experiências prévias do usuário.

3. Considerar os recursos do usuário: as tarefas a serem desempenhadas para a utilização do produto não devem exigir do usuário uma demanda desproporcional de seus recursos.

4. Feedback: garantir que as ações realizadas pelo usuário sejam reconhecidas, além de fornecer informações relevantes sobre os resultados das ações executadas.

5. Prevenção e Recuperação de erros: desenvolver o produto visando à prevenção de erros. Ao ocorrer um erro, não basta que ele seja simplesmente apontado, é fundamental que sejam fornecidas instruções sobre como proceder para que o problema seja resolvido.

6. Controle do usuário: fornecer ao usuário o máximo de controle possível sobre o produto.

7. Clareza/objetividade visual: exibir informações de forma que possam ser de fácil e rápida compreensão. Aspectos como cores, tamanho de imagens e tipografia devem ser levados em conta na disposição do conteúdo.

8. Priorização de funcionalidades e Informações: determinar uma espécie de hierarquia de conteúdos e funções, possibilitando, assim, que o usuário identifique os elementos principais com maior facilidade e rapidez.

9. "Transferência" apropriada de tecnologia: apropriar-se, de maneira coerente e adequada, de tecnologias desenvolvidas para diferentes contextos com o objetivo de aumentar a usabilidade de determinado produto.

10. Clareza: Expressar de maneira clara e objetiva os conteúdos e funcionalidades do produto, bem como garantir que este apresente recomendações sobre suas funcionalidades e modo de utilização.

Complementando, Shneiderman (1998) evidencia "8 regras de ouro" no que tange aos princípios básicos para o design de interfaces: 
1. Consistência: manter um padrão de interface (visual), terminologias e procedimentos para a realização de ações, exigindo assim menor carga de esforço do usuário.

2. Atalhos para usuários frequentes: disponibilizar aos usuários mais experientes meios de executar tarefas de uma forma mais simples e ágil.

3. Oferecer feedback informativo: as tarefas realizadas devem retornar algum tipo de resposta ao usuário, indicando o resultado de suas ações.

4. Apresentar diálogos que indiquem término de uma ação: agrupar e indicar de maneira clara o início, meio e fim de uma sequência de ações necessárias para se executar determinada tarefa.

5. Oferecer prevenção e tratamento de erros: projetar de maneira que a probabilidade de o usuário cometer erros graves seja diminuta. Caso o usuário cometa algum tipo de erro, oferecer instruções e opções claras e objetivas para que seja possível reparar o problema.

6. Permitir reversão de ações de maneira fácil: oferecer a possibilidade de se desfazer ações realizadas. Isso faz com que o usuário sinta-se mais seguro para explorar o sistema/interface.

7. Controle do usuário: possibilitar que o usuário assuma um papel ativo perante a interface, não o restringindo a responder a ações, e sim iniciá-las.

8. Reduzir a carga de memorização: o humano possui capacidade de retenção de memória de curto prazo limitada. Por isso, faz-se necessário que o sistema seja desenvolvido de forma que não se exija do usuário grande carga de esforço para memorização.

Rogers, Sharp e Preece (2013) destacam que existem vários princípios de design relacionados à usabilidade e apontam como sendo os mais "comuns": visibilidade, feedback, restrições, consistência e affordance.

1. Visibilidade: quanto mais fáceis de visualizar as funções, mais facilmente os usuários entenderão como devem proceder para alcançar seus objetivos dentro da navegação da interface.

2. Feedback: toda a ação realizada pelo usuário deve ter alguma espécie de "efeito-resposta", indicando ao usuário os efeitos de suas ações e auxiliando-o a compreender como proceder posteriormente.

3. Restrições: determinar os tipos de ações/interações permitidas ao usuário em dado momento, controlando, assim, de certa forma, o comportamento do usuário reduzindo suas chances de erro.

4. Consistência: estabelecimento/repetição de um padrão para os elementos e ações da interface. Está diretamente ligada ao nível de esforço de aprendizagem para uso de uma interface. Quanto mais consistente for a interface, mais fácil é o processo para entender como operá-la.

5. Affordance: quando se atribui uma característica a determinado elemento/objeto de maneira a permitir que o usuário saiba como deve 
Tatiana Tissa Kawakami, Rosane Alvares Lunardelli, Fernando Luiz Vechiato

O prontuário eletrônico do paciente na perspectiva das recomendações de usabilidade: proposta de organização da informação

utilizá-lo. Em outras palavras, o affordance funciona como uma espécie de "dica" para o usuário.

No que diz respeito as recomendações do design voltadas à usabilidade e centrados no usuário, Norman (2006) apresenta sete princípios:

1. Usar ao mesmo tempo o conhecimento no mundo e o conhecimento na cabeça: o conhecimento de mundo consiste naquele conhecimento externo, que não está embutido na mente do indivíduo. 0 conhecimento da cabeça refere-se àquele internalizado pelo usuário de determinado produto ou interface. Dessa forma, deve-se fazer uso simultâneo do conhecimento presente no mundo e o conhecimento presente na cabeça do indivíduo.

2. Simplificar a estrutura das tarefas: tornar as decisões e ações do usuário o mais simples possível.

3. Tornar as coisas visíveis: certificar-se de que são disponibilizados, de maneira facilmente identificável, opções de ações e funcionalidades de uma interface, bem como o mostrar de maneira clara os resultados das ações tomadas.

4. Fazer corretamente os mapeamentos: os mapeamentos consistem na relação entre os controles disponibilizados ao usuário e os resultados obtidos por meio deles. Com a utilização de mapeamentos naturais (ex.: para que um objeto seja deslocado para cima, apontar com o controle remoto para cima), é possível que o usuário compreenda o funcionamento de uma interface com maior rapidez e facilidade.

5. Explorar o poder das coerções naturais e artificiais: coerções são espécies de restrições que levam o usuário a comportar-se e interagir com o produto de maneiras específicas, funcionando, assim, como um recurso para direcionar/orientar o usuário.

6. Projetar para o erro: sempre procurar antecipar todo e qualquer tipo de erro que possa ocorrer. Essa antecipação permite que sejam previstas também maneiras de lidar com o erro cometido.

7. Quando tudo o mais falhar, padronizar: a padronização permite ao usuário que não seja necessário, sempre ao se deparar com um novo produto, ter que aprender a utilizá-lo como se nunca houvesse interagido com uma interface parecida.

Alguns princípios, regras e recomendações listados a partir das abordagens dos autores possuem similaridades, as quais serão evidenciadas e discutidas na próxima seção, que apesenta a análise dos dados e a discussão dos resultados. 
Tatiana Tissa Kawakami, Rosane Alvares Lunardelli, Fernando Luiz Vechiato O prontuário eletrônico do paciente na perspectiva das recomendações de usabilidade: proposta de organização da informação

\section{$5 \quad$ ANÁLISE E DISCUSSÃO}

Diante das diferentes listas de recomendações de usabilidade apresentadas pelos autores abordados nota-se que, durante todo o ciclo da informação no PEP, é importante levar em conta seu contexto e seus usuários. Nessa perspectiva, pensando-se na questão do fluxo e contexto da informação no prontuário em formato eletrônico, bem como seus usuários, cabe resgatar o pensamento de Ranganathan (2009), bibliotecário que propôs as cinco leis da biblioteconomia.

Embora a relação entre as leis da biblioteconomia e a informação registrada em PEP pareça, a priori, bastante improvável, explica-se: ainda que o autor tenha pensado em tais leis voltadas especificamente ao âmbito da biblioteconomia, elas são altamente adaptáveis e aplicáveis a outros contextos. Assim, compreende-se que é possível transportar e adequar essas leis também para o contexto do PEP, conforme apresentado no quadro seguinte:

Quadro 1 - Adaptação das Leis de Ranganathan para o PEP

\begin{tabular}{|l|l|l|}
\hline \multicolumn{1}{|c|}{ Leis de Biblioteconomia } & \multicolumn{1}{c|}{ Leis do PEP } \\
\hline 1 & Livros são para usar & Informações registradas em PEP são para uso \\
\hline 2 & Para cada leitor, seu livro & Para cada paciente, seu prontuário \\
\hline 3 & Para cada livro, seu leitor & Para cada prontuário, seus usuários \\
\hline 4 & Poupe o tempo do leitor & Poupe o tempo do usuário \\
\hline 5 & A biblioteca é um organismo em crescimento & O PEP é um organismo em crescimento \\
\hline
\end{tabular}

Fonte: Elaborado pelos autores

Ainda que seja possível converter as Leis da Biblioteconomia para o contexto do PEP, é necessário destacar uma grande diferença entre a informação em bibliotecas e a informação no PEP: enquanto os livros são, em sua maioria, destinados ao acesso irrestrito, a informação registrada em prontuários é de caráter confidencial e sigiloso. Reconhece-se a existência de bibliotecas privadas, casos em que, por vezes, o acesso ao seu acervo é 
Tatiana Tissa Kawakami, Rosane Alvares Lunardelli, Fernando Luiz Vechiato O prontuário eletrônico do paciente na perspectiva das recomendações de usabilidade: proposta de organização da informação

restrito, no entanto, no caso das informações presentes no PEP, a sigilosidade é uma característica mandatória.

Frente ao cenário apresentado, nota-se que algumas das recomendações elencadas no tópico anterior (Usabilidade) podem ser relacionadas aos itens propostos no Quadro 1. A primeira lei ("Informações registradas em PEP são para uso") engloba as recomendações de controle do usuário sobre o sistema e fornecimento de informações sobre o sistema. As leis dois e três (respectivamente "Para cada paciente, seu prontuário" e "Para cada prontuário, seus usuários") relacionam-se à recomendação acerca da adequação do sistema ao usuário e seu contexto. A quarta lei ("Poupe o tempo do usuário") compreende as recomendações de diminuição de carga de esforço para memorização, prevenção e recuperação de erros, consistência e objetividade.

Diante do exposto, ao se refletir acerca das Leis da Biblioteconomia de Ranganathan e sua adaptabilidade ao contexto do PEP, evidencia-se o fato de que, independentemente do suporte utilizado ou tipo de conteúdo registrado, os cuidados em torno da informação e seus usuários mostram-se indispensáveis.

Ao analisar e contrastar as recomendações formuladas pelos autores abordados no tópico "Usabilidade", percebeu-se a existência de muitos pontos em comum entre as abordagens dos diferentes autores. Partindo-se da observação de tal similaridade, realizou-se o agrupamento das recomendações de Usabilidade de acordo com os seguintes fatores: a) prevenção e recuperação de erros; b) consistência; c) adequação do sistema ao usuário e seu contexto; d) controle do usuário sobre o sistema; e) fornecimento de informações sobre o sistema; f) diminuição de carga de esforço para memorização; g) apresentação visual; h) objetividade.

Com o propósito de facilitar a visualização e o entendimento da relação entre as recomendações de Nielsen (1995), Jordan (1998), Shneiderman (1998), Norman (2006) e Rogers, Sharp e Preece (2013), destaca-se o quadro a seguir: 
Tatiana Tissa Kawakami, Rosane Alvares Lunardelli, Fernando Luiz Vechiato O prontuário eletrônico do paciente na perspectiva das recomendações de usabilidade: proposta de organização da informação

Quadro 2 - Relação entre recomendações de Usabilidade de Nielsen, Jordan, Shneiderman, Norman e Rogers, Sharp e Preece

\begin{tabular}{|c|c|c|c|c|c|}
\hline $\begin{array}{l}\text { FATORES QUE INFLUENCIAM A } \\
\text { USABILIDADE }\end{array}$ & NIELSEN & JORDAN & SHNEIDERMAN | & NORMAN & $\begin{array}{l}\text { ROGERS, } \\
\text { SHARP E } \\
\text { PREECE }\end{array}$ \\
\hline $\begin{array}{c}\text { PREVENCÇÃO E } \\
\text { RECUPERAÇÃO DE ERROS }\end{array}$ & $x$ & $x$ & $x$ & $x$ & \\
\hline CONSISTÊNCIA & $x$ & $x$ & $x$ & $x$ & $x$ \\
\hline $\begin{array}{c}\text { ADEQUAÇÃO DO SISTEMA AO USUÁRIO } \\
\text { E SEU CONTEXTO }\end{array}$ & $x$ & $x$ & $x$ & $x$ & \\
\hline $\begin{array}{l}\text { CONTROLE DO USUÁRIO SOBRE } 0 \\
\text { SISTEMA }\end{array}$ & $x$ & $x$ & $x$ & $x$ & $x$ \\
\hline $\begin{array}{l}\text { FORNECIMENTO DE INFORMAÇÕES } \\
\text { SOBRE O SISTEMA }\end{array}$ & $x$ & $x$ & $x$ & & $x$ \\
\hline $\begin{array}{c}\text { DIMINUICCÃO DE CARGA DE ESFORÇO } \\
\text { PARA MEMORIZAÇÃO }\end{array}$ & $x$ & & $x$ & & \\
\hline APRESENTAÇÃO VISUAL & $x$ & $x$ & & $x$ & $x$ \\
\hline OBJETIVIDADE & & $x$ & & $x$ & \\
\hline
\end{tabular}

Fonte: Elaborado pelos autores

Embora nem todas as recomendações de usabilidade sejam contempladas por todos os pesquisadores citados, importa ressaltar que cada fator citado no Quadro 2 está presente em ao menos duas das listas de recomendações, evidenciando assim a sua relevância. Uma vez identificada a existência de similaridades entre as recomendações dos autores supracitados, considera-se relevante traçar uma relação entre os fatores que influenciam a usabilidade e suas respectivas recomendações. Para tanto, realizou-se a estruturação de quadros que indicam as ideias de cada autor por recomendação de usabilidade. 
Tatiana Tissa Kawakami, Rosane Alvares Lunardelli, Fernando Luiz Vechiato O prontuário eletrônico do paciente na perspectiva das recomendações de usabilidade: proposta de organização da informação

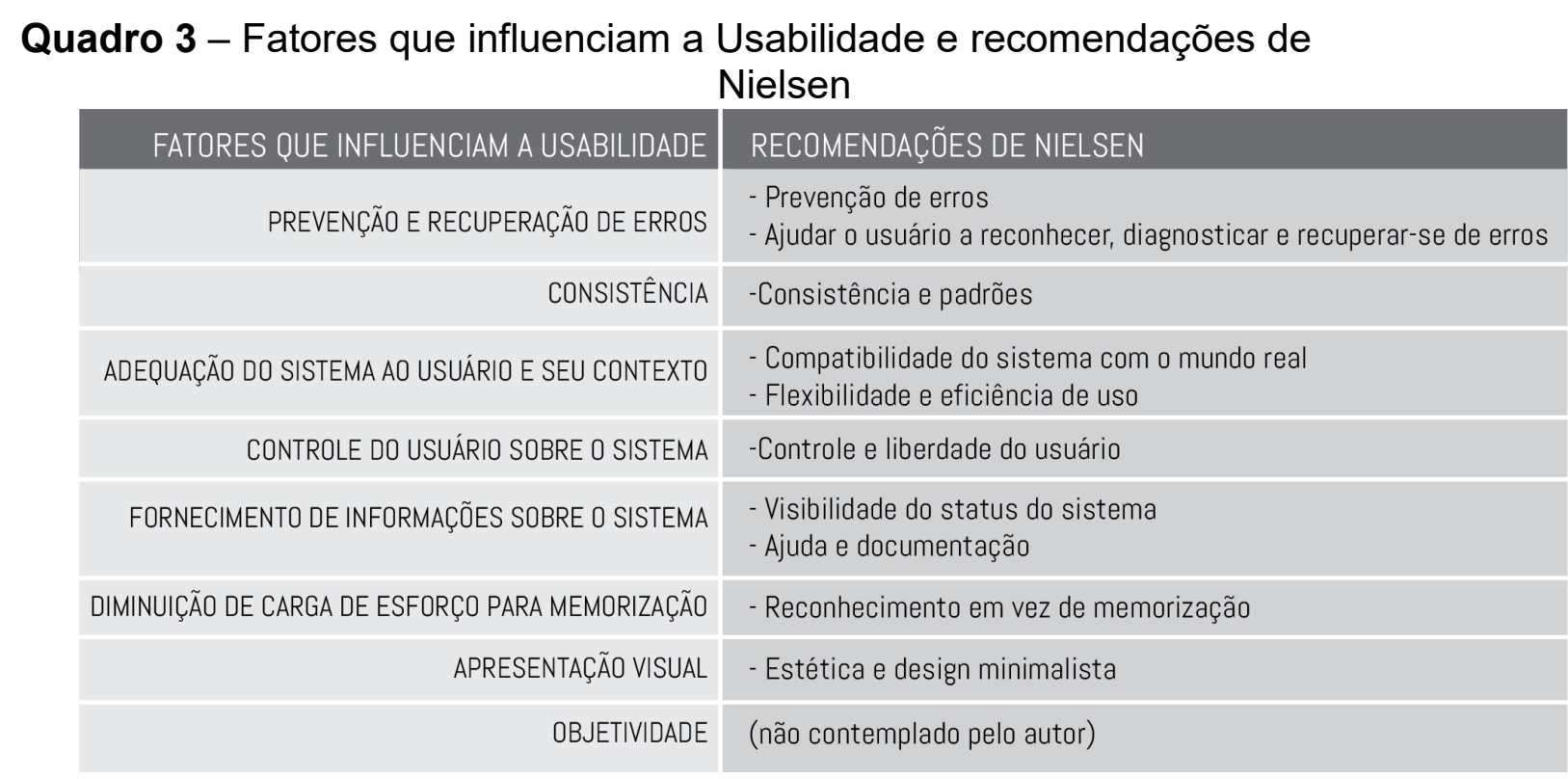

Fonte: Elaborado pelos autores

Com base nas informações presentes no Quadro 3 identifica-se que o único fator não contemplado por Nielsen (1995), o qual realiza pesquisas no âmbito da IHC e Usabilidade, é o da objetividade. Ademais, todos os outros fatores são considerados pelo autor.

\begin{tabular}{|c|c|}
\hline FATORES QUE INFLUENCIAM A USABILIDADE & RECOMENDAÇÕES DE JORDAN \\
\hline PREVENÇÃO E RECUPERAÇÃO DE ERROS & - Prevenção e recuperação de erros \\
\hline CONSISTEENCIA & -Consistência \\
\hline ADEQUAÇÃO DO SISTEMA AO USUÁRIO E SEU CONTEXTO & $\begin{array}{l}\text { - Compatibilidade } \\
\text { - Considerar os recursos do usuário } \\
\text { - "Transferência" apropriada de tecnologia }\end{array}$ \\
\hline CONTROLE DO USUÁRIO SOBRE O SISTEMA & -Controle do usuário \\
\hline FORNECIMENTO DE INFORMAÇÕES SOBRE 0 SISTEMA & - Feedback \\
\hline DIMINUIÇÃO DE CARGA DE ESFORÇO PARA MEMORIZAÇÃO & (não contemplado pelo autor) \\
\hline APRESENTAÇÃO VISUAL & - Clareza/objetividade visual \\
\hline OBJETIVIDADE & $\begin{array}{l}\text { - Priorização de funcionalidades e Informações } \\
\text { - Clareza }\end{array}$ \\
\hline
\end{tabular}

Fonte: Elaborado pelos autores

Ao se analisar o Quadro 4 é possível notar que Jordan (1998), assim como Nielsen (1995), também deixa de elencar somente um dos fatores. Inf. Inf., Londrina, v. 22, n. 3, p. 456 - 483, set./out. 2017. http:www.uel.br/revistas/informacao/ 
Tatiana Tissa Kawakami, Rosane Alvares Lunardelli, Fernando Luiz Vechiato O prontuário eletrônico do paciente na perspectiva das recomendações de usabilidade: proposta de organização da informação

Apesar disso, cabe destacar que, enquanto o segundo deixa de contemplar o fator objetividade, o primeiro, por outro lado, não inclui entre suas recomendações o fator da diminuição de carga de esforço para memorização.

\section{Quadro 5 - Fatores que influenciam a Usabilidade e recomendações de Shneiderman}

\begin{tabular}{|c|c|}
\hline & \\
\hline FATORES QUE INFLUENCIAM A USABILIDADE & RECOMENDAÇÕES DE SHNEIDERMAN \\
\hline PREVENÇÃO E RECUPERAÇÃO DE ERROS & - Oferecer prevenção e tratamento de erros \\
\hline CONSISTÊNCIA & -Consistência \\
\hline ADEQUAÇÃO DO SISTEMA AO USUÁRIO E SEU CONTEXTO & - Atalhos para usuários frequentes \\
\hline CONTROLE DO USUÁRIO SOBRE O SISTEMA & $\begin{array}{l}\text { - Permitir reversão de ações de maneira fácil } \\
\text { - Controle do usuário }\end{array}$ \\
\hline FORNECIMENTO DE INFORMAÇÕES SOBRE O SISTEMA & $\begin{array}{l}\text { - Oferecer feedback informativo } \\
\text { - Apresentar diálogos que indiquem término de uma ação }\end{array}$ \\
\hline DIMINUIÇÃO DE CARGA DE ESFORÇO PARA MEMORIZAÇÃO & - Reduzir a carga de memorização \\
\hline APRESENTAÇÃO VISUAL & (não contemplado pelo autor) \\
\hline OBJETIVIDADE & (não contemplado pelo autor) \\
\hline
\end{tabular}

Fonte: Elaborado pelos autores

Diferente do observado nos quadros com base em Nielsen (1995) e Jordan (1998), casos em que ambos deixam de apontar somente um dos fatores, o Quadro 5, baseado em Shneiderman (1998), suprime dois fatores: apresentação visual e objetividade-

Quadro 6 - Fatores que influenciam a Usabilidade e recomendações de Norman

\begin{tabular}{|r|l|}
\hline FATORES QUE INFLUENCIAM A USABILIDADE & RECOMENDAÇÕES DE NORMAN \\
\hline PREVENÇÃO E RECUPERAÇÃO DE ERROS & - Projetar para o erro \\
\hline CONSISTÊNCIA & - Quando tudo o mais falhar, padronizar \\
\hline ADEQUAÇÃO DO SISTEMA AO USUÁRIO E SEU CONTEXTO & $\begin{array}{l}\text { - Usar ao mesmo tempo o conhecimento no mundo e o conhecimento } \\
\text { na cabeça } \\
\text { - Fazer corretamente os mapeamentos }\end{array}$ \\
\hline CONTROLE DO USUÁRIO SOBRE O SISTEMA & - Explorar o poder das coerções naturais e artificiais \\
\hline DIMINUIÇÃO DE CARGA DE ESFORÇO PARA MEMORIZAÇÃO & (não contemplado pelo autor) \\
\hline APRESENTAÇÃO VISUAL & - Tornar as coisas visíveis \\
\hline OBJETIVIDADE & - Simplificar a estrutura das tarefas \\
\hline
\end{tabular}

Fonte: Elaborado pelos autores 
Tatiana Tissa Kawakami, Rosane Alvares Lunardelli, Fernando Luiz Vechiato O prontuário eletrônico do paciente na perspectiva das recomendações de usabilidade: proposta de organização da informação

Ao se verificar o disposto no Quadro 6, concernente aos fatores que influenciam a Usabilidade de acordo com as recomendações de Norman (2006), nota-se que nesse ocorre a supressão de dois fatores: diminuição de carga de esforço para memorização (que já havia sofrido supressão no Quadro 4) e fornecimento de informações sobre o sistema (elemento que até então era apresentado em todos os quadros).

Quadro 7 - Fatores que influenciam a Usabilidade e recomendações de
Rogers, Sharp e Preece
\begin{tabular}{r|r|r|}
\hline FATORES QUE INFLUENCIAM A USABILIDADE & RECOMENDAÇÕES DE ROGERS, SHARP E PREECE \\
\hline PREVENÇÃO E RECUPERAÇÃO DE ERROS & (não contemplado pelos autores) \\
\hline CONSISTÊNCIA & -Consistência \\
\hline ADEQUAÇÃO DO SISTEMA AO USUÁRIO E SEU CONTEXTO & (não contemplado pelos autores) \\
\hline CONTROLE DO USUÁRIO SOBRE O SISTEMA & - Restrições \\
\hline FORNECIMENTO DE INFORMAÇÕES SOBRE O SISTEMA & - Feedback \\
\hline DIMINUIÇÃO DE CARGA DE ESFORÇO PARA MEMORIZAÇÃOO & (não contemplado pelos autores) \\
\hline APRESENTAÇÃO VISUAL & - Visibilidade \\
\hline OBJETIVIDADE & (não contemplado pelos autores) \\
\hline
\end{tabular}

Fonte: Elaborado pelos autores.

De acordo com o conteúdo do Quadro 7, o qual toma por base as recomendações de Usabilidade de Rogers, Sharp e Preece (2013), observa-se que há quatro fatores não apresentados, sendo esses: prevenção e recuperação de erros; adequação do sistema ao usuário e seu contexto; diminuição de carga de esforço para memorização; objetividade.

Ao se cotejar todos os quadros com recomendações, nota-se que a perspectiva de um autor pode ser complementada pelo pensamento de outro e vice-versa, isso porque as ideias apresentadas por um, por vezes, não figuram entre as recomendações de outro. Dessa forma, acredita-se que a concatenação das recomendações de Nielsen (1995), Jordan (1998), Shneiderman (1998), Norman (2006) e Rogers, Sharp e Preece (2013) possam resultar em uma perspectiva de maior completude, possibilitando assim maiores avanços no sentido de uma interface/produto com maior grau de usabilidade. 
Tatiana Tissa Kawakami, Rosane Alvares Lunardelli, Fernando Luiz Vechiato O prontuário eletrônico do paciente na perspectiva das recomendações de usabilidade: proposta de organização da informação

Uma vez observadas e analisadas as listas de recomendações de Usabilidade propostas pelos autores mencionados, nota-se que apenas Jordan (1998) traz de forma explícita a questão da informação e sua priorização. No quadro 4 é possível notar que o pesquisador apresenta como item de sua lista de recomendações, no fator relacionado à objetividade, o enunciado "Priorização de funcionalidades e Informações", que consiste em antepor funcionalidades e conteúdos de maior relevância, visando assim que esses sejam facilmente identificados.

O fato de Jordan (1998) ser o único a tratar diretamente a questão informacional em um de seus enunciados de recomendação de usabilidade faz com que se evidencie a carência de estudos que levem em conta seus aspectos informacionais.

Nesse sentido, considerando-se a necessidade de maior atenção em torno da questão informacional no que tange aos aspectos relacionados à usabilidade, traz-se à luz as contribuições de Morville e Rosenfeld (2006), que realizam estudos no campo da Al com foco na informação disponibilizada em meio digital. Os autores apresentam como componentes básicos da Al cinco categorias: a) sistemas de organização; b) sistemas de navegação; c) sistemas de busca; d) sistemas de rotulagem; e) sistemas de representação (tesauros, metadados e vocabulários controlados).

Embora todas as categorias elencadas sejam fundamentais no que diz respeito ao profícuo uso da informação, dentre os itens apresentados destacase no presente estudo a questão dos sistemas de organização da informação. A consolidação dos sistemas de organização se dá, de acordo com Morville e Rosenfeld (2006), por meio de esquemas de organização exata e/ou esquemas de organização ambíguos. Ambos os tipos de esquema, por sua vez, apresentam "subesquemas" de organização.

Os esquemas de organização exata são aqueles que se baseiam em moldes bem definidos (exatos) e que não recorrem ao caráter de subjetividade da informação. Dentre os esquemas exatos destacam-se: esquema alfabético; esquema cronológico; esquema geográfico. Já os esquemas ambíguos apresentam-se como de maior complexidade, pois se baseiam na subjetividade 
Tatiana Tissa Kawakami, Rosane Alvares Lunardelli, Fernando Luiz Vechiato O prontuário eletrônico do paciente na perspectiva das recomendações de usabilidade: proposta de organização da informação

do pensamento humano, sendo assim mais difícil organizá-los e mantê-los. Embora sejam complexos, os esquemas ambíguos são amplamente utilizados pelo ser humano, isto porque o indivíduo nem sempre sabe exatamente o que está buscando.

No que tange aos esquemas ambíguos de informação, evidenciam-se os esquemas apresentados por Morville e Rosenfeld (2006), os quais são orientados pelas seguintes classificações: a) assunto/tópico - análise e separação de conteúdos de acordo com os assuntos abordados; b) tarefa esquemas que priorizam processos, funções e tarefas a serem realizadas; $c$ ) público - utiliza esquemas orientados a um grupo específico de usuários; d) metáfora - pautada em esquemas de organização em que se vale do recurso de metáforas; e) híbrido - faz o uso de mais de um tipo de esquema de organização.

Ao se utilizar esquemas híbridos é necessária muita cautela, uma vez que a existência de diferentes lógicas de organização em uma mesma interface dificulta a identificação de um "padrão de tratamento das informações", podendo assim confundir o usuário.

Nesse sentido, referindo-se ao sistema de organização de informações em PEP, importa destacar que este se configura como de altíssima complexidade, uma vez que o prontuário em meio eletrônico caracteriza-se como um esquema de organização híbrido. Ou seja: que faz tanto uso de esquemas exatos (ex.: a ordem cronológica de registro de datas em que foram realizadas consultas), como também de esquemas ambíguos (ex.: disponibilização de conteúdos categorizados por assuntos, tais como ficha de dados pessoais do paciente).

Assim, ao se analisar os conteúdos registrados em PEP sob a perspectiva dos sistemas de organização propostos por Morville e Rosenfeld (2006), evidencia-se a natureza intrincada dos conteúdos registrados nesse tipo de documento. Além disso, destaca-se o imperativo no que tange ao seu aspecto de facilidade de uso. Dessa forma, coloca-se em foco a questão da Usabilidade, levando-se em conta seus aspectos informacionais, no prontuário em versão eletrônica. 
Tatiana Tissa Kawakami, Rosane Alvares Lunardelli, Fernando Luiz Vechiato O prontuário eletrônico do paciente na perspectiva das recomendações de usabilidade: proposta de organização da informação

Nessa direção, retomando-se as recomendações de usabilidade elencadas com base em Nielsen (1995), Jordan (1998), Shneiderman (1998), Norman (2006) e Rogers, Sharp e Preece (2013), as quais predominantemente concernem à usabilidade de interfaces, e combinando-as com as reflexões de Morville e Rosenfeld (2006) e Ranganathan (2009), propõe-se a adaptação dessas recomendações para o PEP, levando-se em conta seus aspectos informacionais. Nesse sentido, desenvolveu-se o quadro a seguir, o qual apresenta um checklist com recomendações de Usabilidade para o PEP. 
Tatiana Tissa Kawakami, Rosane Alvares Lunardelli, Fernando Luiz Vechiato

O prontuário eletrônico do paciente na perspectiva das recomendações de

usabilidade: proposta de organização da informação

\section{Quadro 8 - Checklist com recomendação de Usabilidade para o PEP}

\begin{tabular}{|c|c|c|}
\hline $\begin{array}{l}\text { FATORES QUE } \\
\text { INFLUENCIAM A } \\
\text { USABILIDADE }\end{array}$ & $\begin{array}{l}\text { RECOMENDAÇ̃̃ES GERAIS } \\
\text { DE USABILIDADE PARA O PEP }\end{array}$ & $\begin{array}{l}\text { RECOMENDAÇÕES DE USABILIDADE PARA O PEP } \\
\text { COM ENFOQUE NO ASPECTO INFORMACIONAL }\end{array}$ \\
\hline$\square$ CONSISTÊNCIA & $\begin{array}{l}\text { Manter padrão de elementos visuais da interface do } \\
\text { PEP. Manter padrão lógico para realização de tarefas } \\
\text { (ex.: tarefas similares devem ser ativadas por meio } \\
\text { de comandos similares). }\end{array}$ & $\begin{array}{l}\text { Padronização de termos e esquemas de organização } \\
\text { da informação. }\end{array}$ \\
\hline $\begin{array}{l}\square \\
\text { ADEQUAÇÃO DO } \\
\text { SISTEMA AO } \\
\text { USUÁRIO E SEU } \\
\text { CONTEXTO }\end{array}$ & $\begin{array}{l}\text { Levar em conta especificidades dos usuários do PEP, } \\
\text { bem como o seu contexto (ex.: a apresentação de } \\
\text { muitas colunas para usuários que utilizam monitor de } \\
\text { baixa resolução não é adequada). }\end{array}$ & $\begin{array}{l}\text { Adoção de linguagem (termos) compativel ao contexto } \\
\text { dos profissionais da equipe de cuidados com a saúde e } \\
\text { demais profissionais envolvidos na utilização do PEP. } \\
\text { Trabalhar de maneira articulada com as diferentes } \\
\text { categorias de profissionais que utilizam o PEP para que } \\
\text { seja possível alinhar os termos utilizados uma vez que, } \\
\text { por mais importante que seja adotar termos } \\
\text { reconhecidos pelos usuários, é igualmente relevante } \\
\text { que todos os profissionais façam uso das mesmas } \\
\text { terminologias, evitando assim falhas de comunicação. }\end{array}$ \\
\hline $\begin{array}{l}\square \\
\text { PREVENÇÃO E } \\
\text { RECUPERAÇÃO DE } \\
\text { ERROS }\end{array}$ & $\begin{array}{l}\text { Proporcionar mecanismos para que o usuário possa } \\
\text { reconhecer, compreender e recuperar-se de erros. }\end{array}$ & $\begin{array}{l}\text { Apresentação de instruções sobre funcionalidades e } \\
\text { ações para realização de determinada tarefa. } \\
\text { Quando ocorrer um erro, apresentar informação } \\
\text { especificando qual foi o erro e descrever opções para } \\
\text { que o usuário consiga se recuperar do erro. }\end{array}$ \\
\hline $\begin{array}{l}\square \\
\text { CONTROLE DO } \\
\text { USUÁRIO SOBRE O } \\
\text { SISTEMA }\end{array}$ & $\begin{array}{l}\text { Atribuir ao usuário do PEP opções que possibilitem a } \\
\text { execução de tarefas com autonomia. Fornecer ao } \\
\text { usuário, na medida do possível, opção de } \\
\text { reverter/desfazer ações. } \\
\text { Não atribuir ao usuário capacidade de ações que } \\
\text { modifiquem permanentemente o sistema (ex.: deletar } \\
\text { um arquivo que não poderá ser recuperado } \\
\text { posteriormente). } \\
\text { Oferecer instruções detalhadas para usuários menos } \\
\text { experientes. } \\
\text { Oferecer atalhos para agilizar o trabalho de usuários } \\
\text { mais experientes. }\end{array}$ & $\begin{array}{l}\text { Permitir que o usuário do PEP tenha controle sobre as } \\
\text { informações registradas, porém sem comprometer a } \\
\text { integridade e legitimidade do documento (ex.: mesmo } \\
\text { permitindo que o usuário realize alterações de conteúdo } \\
\text { registrado no PEP, manter um histórico das edições } \\
\text { realizadas e seus respectivos autores). }\end{array}$ \\
\hline $\begin{array}{l}\square \\
\text { FORNECIMENTO DE } \\
\text { INFORMAÇÕES } \\
\text { SOBRE O SISTEMA }\end{array}$ & $\begin{array}{l}\text { Apresentar feedback ao usuário indicando o status } \\
\text { do sistema (ex.: exibir mensagem indicando que a } \\
\text { inserção de um texto foi salva no sistema). }\end{array}$ & $\begin{array}{l}\text { Disponibilizar para usuários do PEP manual que } \\
\text { apresente instruções sobre o sistema (como utilizá-lo). } \\
\text { O software de PEP deve sempre fornecer ao seu } \\
\text { usuário um posicionamento sobre seu status. } \\
\text { Disponibilizar informação indicando término de ação. }\end{array}$ \\
\hline $\begin{array}{l}\square \\
\text { DIMINUIÇÃO DE } \\
\text { CARGA DE ESFORÇO } \\
\text { PARA MEMORIZAÇÃO }\end{array}$ & $\begin{array}{l}\text { Não exigir que o usuário decore funcionalidades ou } \\
\text { informações do sistema. } \\
\text { Sempre disponibilizar na interface as ações e opções } \\
\text { possiveis. }\end{array}$ & $\begin{array}{l}\text { Valer-se da repetição de informações em diferentes } \\
\text { telas da interface, poupando assim o usuário da } \\
\text { necessidade de memorização de conteúdos. } \\
\text { Garantir que o material de instruções sobre o sistema } \\
\text { seja de fácil acesso ao usuário (para casos em que o } \\
\text { usuário esqueça-se, por exemplo, de como executar } \\
\text { determinada tarefa). }\end{array}$ \\
\hline$\square$ OBJETIVIDADE & $\begin{array}{l}\text { Trabalhar a estrutura e funcionalidades do PEP para } \\
\text { que essas sejam simples, facilitando assim sua } \\
\text { utilização. }\end{array}$ & $\begin{array}{l}\text { Priorizar informações relevantes. } \\
\text { A informação deve ser organizada de maneira objetiva } \\
\text { e lógica. Para tanto, indica-se a utilização de Sistemas } \\
\text { de Organização da Informação*. }\end{array}$ \\
\hline $\begin{array}{l}\square \\
\text { APRESENTAÇÃO } \\
\text { VISUAL }\end{array}$ & $\begin{array}{l}\text { Utilizar linguagem visual adequada que propicie a } \\
\text { comunicação de maneira clara e direta, permitindo } \\
\text { assim a visualização de conteúdos. }\end{array}$ & $\begin{array}{l}\text { Priorizar a visualização de informações de maior } \\
\text { relevância. } \\
\text { Realizar criterioso agrupamento de informações, com } \\
\text { base em Sistemas de Organização da Informação*, e } \\
\text { traçar conexão visual (por meio de cores, formas, } \\
\text { tipografia e ícones) entre conteúdos correlatos. }\end{array}$ \\
\hline \multicolumn{3}{|c|}{$\begin{array}{l}\text { * Os Sistemas de Organização da Informação podem se dar por meio de esquemas exatos ou esquemas ambíguos. Os esquemas exatos } \\
\text { podem ser alfabéticos, cronológicos ou geográficos. Já os esquemas ambíguos podem se dar por: assunto/tópico, tarefa, público, metáfora } \\
\text { ou, por último, híbrido. Esquemas híbridos são aqueles que fazem uso de mais de um tipo de esquema de organização. }\end{array}$} \\
\hline
\end{tabular}

Fonte: Elaborado pelos autores

Inf. Inf., Londrina, v. 22, n. 3, p. 456 - 483, set./out. 2017. http:www.uel.br/revistas/informacao/ 
Tatiana Tissa Kawakami, Rosane Alvares Lunardelli, Fernando Luiz Vechiato O prontuário eletrônico do paciente na perspectiva das recomendações de usabilidade: proposta de organização da informação

Espera-se que o checklist apresentado, o qual foi desenvolvido com base na combinação e articulação de conteúdos que versam acerca da Usabilidade e Organização da Informação, possa contribuir para o aprimoramento do PEP, possibilitando assim maior eficiência e eficácia em seu uso.

\section{CONSIDERAÇÕES FINAIS}

A informação é elemento ubíquo e essencial em qualquer esfera da vida em sociedade. No âmbito da Saúde não é diferente. A informação traduz-se como fator determinante para que equipes de instituições de cuidados com a Saúde possam desempenhar seu trabalho de maneira adequada. O processo de tratamento de enfermos inevitavelmente gera informações diversas, tais como histórico de medicamentos administrados, antecedentes pessoais, notas de evolução, relatórios de imagens e relatórios laboratoriais, entre outros.

Nessa direção, reconhecendo-se a informação como complexo elemento nuclear para o desenvolvimento do trabalho de profissionais da área da Saúde, fez-se relevante tecer investigações em torno de sua forma de organização, buscando assim otimizar o seu uso. Dessa forma, colocou-se em evidência o prontuário do paciente, o qual consiste em documento depositário de informações concernentes ao processo de cuidados com a Saúde de um paciente. Além de sua indiscutível função no auxílio de cuidados com o paciente, o prontuário também se apresenta como fonte de informação para o desenvolvimento de estudos tanto na esfera clínica quanto na esfera acadêmica. Outra função assumida por esse dossiê está relacionada ao seu valor jurídico, podendo ser utilizado como documento comprobatório.

O prontuário foi utilizado por um longo período exclusivamente em suporte de papel, porém, com o advento de novas tecnologias, apresentou-se a possibilidade de transportá-lo para o formato eletrônico, passando assim a existir o PEP. O prontuário em suporte eletrônico apresenta diversas vantagens em relação ao prontuário de papel, dentre elas: maior facilidade na recuperação de informações por meio de sistema de busca; diminuição da probabilidade de registro duplicado de informações, entre outros. 
Tatiana Tissa Kawakami, Rosane Alvares Lunardelli, Fernando Luiz Vechiato O prontuário eletrônico do paciente na perspectiva das recomendações de usabilidade: proposta de organização da informação

Ainda que haja benefícios na utilização do PEP, deve-se reconhecer também os obstáculos e problemas a serem enfrentados em decorrência de sua utilização. O uso do prontuário em formato eletrônico exige certas condições como: infraestrutura adequada (hardware e energia elétrica); alto investimento de tempo e capital para sua implantação e manutenção; colaboração da equipe de usuários no que se refere ao treinamento de sua utilização. Dentre os fatores citados, destaca-se o aspecto da colaboração dos usuários no processo de implantação e utilização do PEP. É determinante que a equipe que utilizará essa ferramenta esteja integrada e inteirada no que tange ao potencial do prontuário eletrônico como instrumento catalizador do processo de cuidados com a Saúde.

Dessa forma, diante do referencial teórico construído ao longo do estudo, compreende-se que para que os usuários encarem o PEP de maneira positiva, aumentando assim a chance de sucesso em sua implantação e uso, faz-se pertinente a questão da usabilidade. Em síntese, a usabilidade refere-se ao grau de facilidade de uso de determinado objeto/produto. Nesse sentido, realizou-se o estudo de recomendações gerais de Usabilidade para, a partir disso, delinear uma proposta de recomendações de usabilidade para o PEP.

Expressiva parte das recomendações de Usabilidade elencadas pelos autores consultados - Nielsen (1995), Jordan (1998), Shneiderman (1998), Rogers, Sharp e Preece (2013) e Norman (2006) - referem-se a aspectos operacionais e visuais da interface, não contemplando de maneira clara e direta a questão informacional. Nesse sentido, fez-se uso das perspectivas de Morville e Rosenfeld (2006) e Ranganathan (2009) trabalhando-as com enfoque na informação.

Assim, com base na inserção de considerações acerca da informação como elemento influenciador no processo, apresentou-se um checklist com recomendações de usabilidade para o PEP. Espera-se que por meio desse instrumento seja possível contribuir para que desenvolvedores e usuários do prontuário possam avaliar sua usabilidade, propiciando sua melhoria e buscando assim maiores chances de sucesso na implantação e utilização do PEP. 
Tatiana Tissa Kawakami, Rosane Alvares Lunardelli, Fernando Luiz Vechiato O prontuário eletrônico do paciente na perspectiva das recomendações de usabilidade: proposta de organização da informação

Além disso, o estudo destacou a relação entre usabilidade e informação, abrindo-se caminhos para novos estudos que possam investigar a questão da própria "Usabilidade da informação" no PEP.

A abordagem interdisciplinar empregada na pesquisa, a interlocução entre diferentes campos do conhecimento (Usabilidade, Ergonomia, IHC, $\mathrm{Al}$ e Design), ratificou a capacidade da $\mathrm{Cl}$ de instituir-se como campo de pesquisa capaz de contribuir para a investigação e solução de problemas de outras áreas de estudos. Nesse caso, a Área, por meio de sua linha de pesquisa acerca da Organização da Informação, auxiliou na construção de novas perspectivas no que tange a estruturação do PEP e seus conteúdos, contribuindo assim para o universo da informação na área da Saúde.

\section{REFERÊNCIAS}

BATLEY, S. Information architecture for information professionals. Oxford: Chandos Publishing, 2007.

BENTES PINTO, V. Prontuário eletrônico do paciente: documento técnico de informação e comunicação do domínio da saúde. Encontros Bibli: Revista Eletrônica de Biblioteconomia e Ciência da Informação, Florianópolis, v. 17, n. 21, p. 34-48, 2006. Disponível em: <http://www.redalyc.org/articulo.oa?id=14702104> Acesso em: 10 jan. 2014.

CAMARGO, L. S. A; VIDOTTI, S. A. B. G. Arquitetura da Informação: uma abordagem prática para o tratamento de conteúdo e interface em ambientes informacionais digitais. Rio de Janeiro: LTC, 2011.

CANÊO, P. K.; RONDINA, J. M. Prontuário eletrônico do paciente: conhecendo as experiências de sua implantação. Journal of Health Informatics, São Paulo, v. 6, n. 2, 2014. Disponível em: <http://www.jhi-sbis.saude.ws/ojsihi/index.php/jhi-sbis/article/view/289/197>. Acesso em: 2 mar. 2015.

CARDOSO, A. M. P. Pós-modernismo e informação: conceitos complementares? Perspectivas e $\mathbf{m}$ Ciência da Informação, Belo Horizonte, v. 1, n. 1, p. 63-79, jan./jun. 1996. Disponível em: <http://www.brapci.inf.br/ repositorio/2010/08/pdf 44afe65e85 0011622.pdf>. Acesso em: 14 dez. 2013. 
Tatiana Tissa Kawakami, Rosane Alvares Lunardelli, Fernando Luiz Vechiato O prontuário eletrônico do paciente na perspectiva das recomendações de usabilidade: proposta de organização da informação

CONSELHO REGIONAL DE MEDICINA DO DISTRITO FEDERAL. Prontuário médico do paciente: guia para uso prático. Brasília: Conselho Regional de medicina, 2006. Disponível em:

$<$ http://www.periciamedicadf.com.br/publicacoes/prontuario medico paciente.p df $>$. Acesso em: 12 fev. 2014.

CYBIS, W.; BETIOL, A. H.; FAUST, R. Ergonomia e usabilidade:

conhecimentos, métodos e aplicações. 2. ed. São Paulo: Novatec, 2010.

GALVÃO, M. C. B.; RICARTE, I. L. M. O prontuário eletrônico do paciente no século XXI: contribuições necessárias da ciência da informação. InCID:

Revista de Ciência da Informação e Documentação, Brasil, v. 2, n. 2, p. 77 100, dez. 2011. Disponível em:

<http://www.revistas.usp.br/incid/article/view/42353/46024>. Acesso em: 21 abr. 2014.

. Prontuário do paciente. Rio de Janeiro: Guanabara Koogan, 2012.

GIL, A. C. Como elaborar projetos de pesquisa. 4. ed. São Paulo: Atlas, 2008.

GONZALEZ DE GOMEZ, M. N. O domínio das informações em saúde. In: BENTES PINTO, V.; CAMPOS, H. H. (Org.). Diálogos paradigmáticos sobre informação para a área de saúde. Fortaleza: Edições UFC, 2013. p. 29-64.

JENAL, S.; ÉVORA, Y. D. M. Revisão de literatura: implantação de prontuário eletrônico do paciente. Journal of Health Informatics, São Paulo, v. 4, n. 4, p. 176-181, out./dez. 2012. Disponível em: <http://www.jhi-sbis.saude.ws/ojsihi/index.php/jhi-sbis/article/view/216/141>. Acesso em: 2 fev. 2014.

JORDAN, P. W. An introduction to usability. Padstow: CRC Press, 1998.

LUNARDELLI, R. S. A.; MOLINA, L. G.; ALVES, R. O. B. Sistemas de informação na área da saúde: em destaque o prontuário eletrônico do paciente no Hospital do Câncer de Londrina. In: DUARTE, Z.; FARIAS, L. (Org.). A medicina na era da informação. Bahia: EDUFBA, 2009. p. 489-502.

LUNARDELLI, R. S. A.; TONELLO, I. M. S.; MOLINA, L. G. A constituição da memória dos procedimentos em saúde no contexto do prontuário eletrônico do paciente. Informação \& Informação, Londrina, v. 19, n. 3, p. 107-124, set./dez. 2014. Disponível em:

<http://www.uel.br/revistas/uel/index.php/informacao/article/view/18837>.

Acesso em: 25 mar. 2014.

MASSAD, E. et al. O prontuário eletrônico do paciente na assistência, informação e conhecimento médico. São Paulo: H. de F. Marin, 2003. Disponível em: $<$ http://www.sbis.org.br/site/arquivos/prontuario.pdf> . Acesso em: 10 out. 2013. 
Tatiana Tissa Kawakami, Rosane Alvares Lunardelli, Fernando Luiz Vechiato

O prontuário eletrônico do paciente na perspectiva das recomendações de usabilidade: proposta de organização da informação

MORVILLE, P.; ROSENFELD, L. Information archietecture for the world wide web. 3. ed. Sebastopol: O'Reily Media, 2006.

NIELSEN, J. Ten usability heuristics. 1995. Disponível em: $<$ http://www.useit.com/papers/heuristic/heuristic list.html>. Acesso em: 27 out. 2013.

NORMAN, D. A. O design do dia-a-dia. Trad.de Ana Deiró. Rio de Janeiro: Rocco, 2006.

RANGANATHAN, S. R. As cinco leis da biblioteconomia. Trad. de Tarcisio Zandonade. Brasília: Briquet de Lemos, 2009.

SANTOS, I. E. Manual de métodos e técnicas de pesquisa científica. 5. ed. Niterói: Impetus, 2005.

ROGERS, Y.; SHARP, H.; PREECE, J. Design de interação: além da interação humano-computador. Trad. de Isabela Gasparini. 3. ed. Porto Alegre: Bookman, 2013.

SHNEIDERMAN, B. Designing the user interface: strategies for effective human-computer-interaction. 3. ed. EUA: Addison Wesley, 1998.

VECHIATO, F. L.; VIDOTTI, S. A. B. G. Usabilidade em ambientes informacionais digitais: fundamentos e avaliação. In: CONGRESSO NACIONAL DE BIBLIOTECÁRIOS, ARQUIVISTAS E DOCUMENTALISTAS (BAD), 11., 2012, Lisboa. Actas... Lisboa: Associação Portuguesa de Bibliotecários, Arquivistas e Documentalistas, 2012. p. 1-10.

WAGER, K. A.; LEE, F. W.; GLASER, J. P. Health care information systems: a practical approach for health care management. 2. ed. São Francisco: Jossey-Bass, 2009.

WEBER, C. A. T. O prontuário médico e a responsabilidade civil. Porto Alegre: EDIPUCRS, 2010.

Title

The organization of information in electronic patient record under the perspective of usability recommendations: proposition of organization of information.

\begin{abstract}
:
Introduction: Among the various areas of studies, health information is highlighted in this study. More specifically, the patient's electronic medical records and issues related to it's informational organization and usability.

Objectives: suggest Usability recommendations applicable to the Electronic Patient Record. More specifically, identify, according to the specialized literature,
\end{abstract}


Tatiana Tissa Kawakami, Rosane Alvares Lunardelli, Fernando Luiz Vechiato

O prontuário eletrônico do paciente na perspectiva das recomendações de usabilidade: proposta de organização da informação

recommendations of Usability, as well as to develop a checklist with recommendations of Usability for the Electronic Patient Record.

Methodology: the study's basic purpose is the theoretical nature. The deductive method of documental delimitation was chosen.

Results: elaboration of checklist with recommendations of Usability for Electronic Patient Records.

Conclusion: Usability recommendations can be used to improve electronic patient records. However, it should be noted that knowledge in the scope of Information Science should be considered and summed up, since a great deal of content related to Usability refers to operational and visual aspects of the interface, not clearly or directly contemplating the issues related to information.

Keywords: Usability. Electronic patient record. Organization of Information.

\section{Titulo}

Registro electrónico del paciente en la perspectiva de las recomendaciones de usabilidad: ponencia de la organización de la información en el

\section{Resumen:}

Introducción: Entre las diversas esferas de estudios existentes, se resalta la información en el área de la Salud. Más específicamente, se enfoca el registro electrónico del paciente y cuestiones relacionadas a su Organización de la Información y Usabilidad.

Objetivos: desarrollar una propuesta de recomendaciones de Usabilidad aplicables al registro electrónico del paciente. Mas especificamente, identificar, a través de revisión de literatura especializada, recomendaciones de Usabilidad y desarrollar un checklist con recomendaciones de Usabilidad para el registro electrónico del paciente.

Metodología: El estudio es de propósito básico y cuño teórico. Se utilizó el método deductivo de delineación documental.

Resultados: elaboración de checklist con recomendaciones de Usabilidad para el registro electrónico del paciente.

Conclusión: las recomendaciones de Usabilidad pueden ser utilizadas para la mejora del registro electrónico del paciente. Sin embargo, se destaca que los conocimientos del ámbito de la Ciencia de la Información deben ser considerados y sumados, ya que gran parte de contenidos relacionados a la Usabilidad se refieren a aspectos operacionales y visuales de la interfaz, no contemplando de manera clara y directa la cuestión de la información.

Palabras clave: Usabilidad. Registro electrónico del paciente. Organización de la Información.

Recebido em: 01.06.2017

Aceito em: 13.12.2017 